\title{
Spatial Diversity and Channel Statistics-Based RF-Baseband Co-Design for Antenna Selection
}

\author{
Pallav Sudarshan, Neelesh B. Mehta, Andreas F. Molisch, Jin Zhang
}

TR2004-077 January 2004

\begin{abstract}
For MIMO systems, we introduce two novel architectures that significantly reduce the required number of expensive RF chains and still perform as well as a conventional full complexity solution for many cases of interest. The architectures we propose combine RF pre-processing - based soley on channel statistics and not instananeous channel state - with selection and corresponding baseband signal processing. For a system with $\mathrm{L}$ RF chains, $\mathrm{Nt}$ transmit and $\mathrm{Nr}$ receive antennas, the first architecture uses an L x Nr RF pre-processing matrix that outputs only $\mathrm{L}$ streams followed by baseband signal processing. The second mone uses an $\mathrm{Nr} \times \mathrm{Nr}$ RF pre-processing matrix followed by selection of $\mathrm{L}$ streams and baseband signal processing. We derive the optimal RF pre-processing matrices and the corresponding baseband combining vectors that maximize the average output signal to noise ratio. A beamforming-based geometric intuition is also developed. With the contraints of tody's IC fabrication technology in mind, an approximation that requires only variable phase-shifters and adders, is also proposed and shown to incur a negligible loss in performance. In the process, a fair benchmark for comparing with the previously proposed FFT Butler preprocessing is provided.
\end{abstract}

IEEE Vehicular Technology Conference (VTC) 2004 Fall

This work may not be copied or reproduced in whole or in part for any commercial purpose. Permission to copy in whole or in part without payment of fee is granted for nonprofit educational and research purposes provided that all such whole or partial copies include the following: a notice that such copying is by permission of Mitsubishi Electric Research Laboratories, Inc.; an acknowledgment of the authors and individual contributions to the work; and all applicable portions of the copyright notice. Copying, reproduction, or republishing for any other purpose shall require a license with payment of fee to Mitsubishi Electric Research Laboratories, Inc. All rights reserved. 

IEEE Vehicular Technology Conference (VTC) 2004 Fall 


\section{Spatial Diversity and Channel Statistics-Based RF-Baseband Co-Design for Antenna Selection}

\author{
Pallav Sudarshan, Student Member, IEEE, ${ }^{\dagger}$ \\ Electrical and Computer Engineering Department, \\ North Carolina State University, \\ Raleigh, NC, USA. \\ psudars@ncsu.edu
}

\author{
Neelesh B. Mehta, Member, IEEE, \\ Andreas F. Molisch, Senior Member, IEEE, \\ Jin Zhang, Member, IEEE, \\ Mitsubishi Electric Research Labs, \\ 201 Broadway, Cambridge, MA, USA. \\ \{mehta, molisch, jzhang\}@merl.com
}

\begin{abstract}
For MIMO systems, we introduce two novel architectures that significantly reduce the required number of expensive RF chains and still perform as well as a conventional full complexity solution for many cases of interest. The architectures we propose combine RF pre-processing - based solely on channel statistics and not instantaneous channel state - with selection and corresponding baseband signal processing. For a system with $L \mathbf{R F}$ chains, $N_{t}$ transmit and $N_{r}$ receive antennas, the first architecture uses an $L \times N_{r}$ RF pre-processing matrix that outputs only $L$ streams followed by baseband signal processing. The second one uses an $N_{r} \times N_{r}$ RF pre-processing matrix followed by selection of $L$ streams and baseband signal processing. We derive the optimal RF pre-processing matrices and the corresponding baseband combining vectors that maximize the average output signal to noise ratio. A beamforming-based geometric intuition is also developed. With the constraints of today's IC fabrication technology in mind, an approximation that requires only variable phase-shifters and adders, is also proposed and shown to incur a negligible loss in performance. In the process, a fair benchmark for comparing with the previously proposed FFT Butler preprocessing is provided.

Index Terms-MIMO systems, diversity methods, antenna arrays, antenna selection, signal to noise ratio, phase shifters, channel statistics, transmitting antennas, receiving antennas, optimization methods
\end{abstract}

\section{INTRODUCTION}

Spatial diversity techniques use multiple antenna elements at the transmitter and receiver to combat fading by increasing the diversity order of the channel [1]. However, the increased hardware and signal processing complexity entailed at the transmitter and the receiver has inhibited the widespread adoption of such multiple input multiple output (MIMO) systems. Antenna selection algorithms, which reduce the hardware complexity by processing only an adaptively chosen subset of the available antennas, have therefore received considerable attention [2], [3]. While antenna selection does not affect the diversity order, a penalty must be paid in the form of a lower beamforming gain [4].

In this paper, we introduce a novel joint design that involves using a RF pre-processing matrix, $M$, followed by selection, if

\footnotetext{
${ }^{\dagger}$ This work was done when the author was at Mitsubishi Electric Research Labs.

${ }^{\ddagger}$ A. F. Molisch is also at the Department of Electroscience, Lund University, Lund, Sweden.
}

necessary, and baseband signal processing. The novelty lies in using only channel statistics, and not the instantaneous channel state, to design the RF pre-processing, and combining it with selection. Other recent approaches based on joint RF-baseband design are known to significantly outperform conventional antenna selection with the same number of RF chains [6], [7]. In [6], the elements of the matrix $M$ were tuned to the instantaneous channel state $\boldsymbol{H}$, and performance close to the full complexity receiver (with more RF chains) was achieved. In [7], an alternate approach, in which $\boldsymbol{M}$ - an FFT Butler matrix - was completely independent of $\boldsymbol{H}$ was investigated. While the gains were promising, they were optimal only for certain fixed angles of arrival (AoA).

The large-scale parameters of the channel vary very slowly compared to the small-scale Rayleigh fading variations. Using only the statistical knowledge and avoiding instantaneous channel-induced variations reduces the higher RF losses and tighter design constraints on the RF elements required in [6]. This also eases the feedback burden about the channel state considerably, when pre-processing is employed at the transmitter. Henceforth, we shall refer to the channel statistics-based solutions as time-invariant solutions. In this paper, we focus on pre-processing and selection at the receiver; analogous arguments hold for the transmitter, as well.

We propose and analyze two different, but related, architectures employing joint RF-baseband design, namely, TI (timeinvariant) and TI-S (time-invariant with selection). Given $L$, the number of demodulator (demod) chains, $N_{t}$, the number of transmit antennas, and $N_{r}$, the number of receive antennas, we first derive the optimal $L \times N_{r}$ RF pre-processing matrix, $M_{\mathrm{TI}}$ that maximizes the average signal to noise ratio (SNR) after baseband processing. This solution eliminates the need for selection altogether. Next, we consider a receiver that combines an $N_{r} \times N_{r}$ pre-processing matrix, $\boldsymbol{M}_{\mathrm{TI}-\mathrm{S}}$, and selection. The optimal $\boldsymbol{M}_{\mathrm{TI}-\mathrm{S}}$ is derived using a technique that successively refines a lower bound on the average SNR. Keeping in mind the practical fabrication constraints that prefer variable phase-shifters as RF elements [8], a phase-only approximation to $\boldsymbol{M}_{\mathrm{TI}}$ and $\boldsymbol{M}_{\mathrm{TI}-\mathrm{S}}$ is also presented and shown to incur a negligible loss in the performance.

Multiple antennas can also be used for spatially multi- 


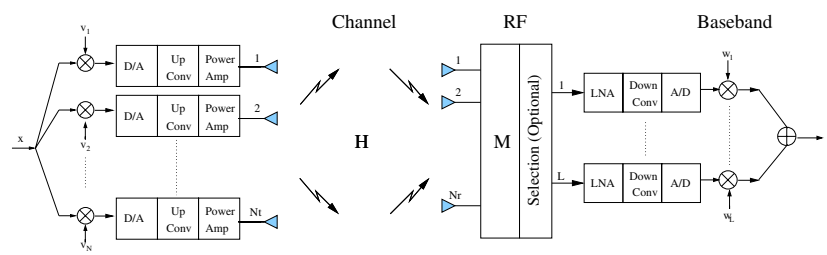

Fig. 1. Block Diagram for Diversity Transmission with RF-Baseband design

plexing multiple data streams. While for spatial diversity, the performance metric is the output SNR, for spatial multiplexing, it is the information theoretic capacity measured in bits/s/Hz. The RF-baseband co-design for spatial multiplexing is investigated in [11].

The outline of this paper is as follows. Section II sets up the spatial diversity system model and the channel model. Sections III and IV derive the optimal $L \times N_{r}$ and $N_{r} \times N_{r}$ structures, respectively. The results are studied in Section V, and our conclusions follow in Section VI.

\section{SySTEM MOdEL}

Consider a MIMO system with $N_{t}$ transmit and $N_{r}$ receive antennas as shown in Fig. 1. Both the transmitter and receiver have complete channel state information (CSI). The same information symbol $x$, weighted by vector $\boldsymbol{v}$, is transmitted from all the antennas. While the transmitter has $N_{t}$ modulator chains, the receiver has $L\left(L \leq N_{r}\right)$ demod chains. The received vector $\boldsymbol{y}$ is given by

$$
\boldsymbol{y}=\sqrt{\frac{\rho}{N_{t}}} \boldsymbol{H} \boldsymbol{v} x+\boldsymbol{n},
$$

where $\boldsymbol{H}$ is $N_{r} \times N_{t}$ channel matrix and $\rho$ is the received SNR input to a receiver's antenna. $\boldsymbol{n}$ is the additive white Gaussian noise and follows the distribution $\mathcal{N}_{c}\left(\mathbf{0}, \boldsymbol{I}_{\boldsymbol{N}_{r}}\right)$, where $\mathcal{N}_{c}$ denotes the complex Gaussian distribution, $\mathbf{0}$ is the all zeros mean vector and $\boldsymbol{I}_{\boldsymbol{N}_{\boldsymbol{r}}}$ is the $N_{r} \times N_{r}$ identity covariance matrix. In maximum ratio transmission (MRT), $\boldsymbol{v}$ is set to $\boldsymbol{v}_{1}$, the right singular vector of the largest singular value of $\boldsymbol{H}$. Given that only modifications to a maximum ratio combining receiver are considered in this paper, $\boldsymbol{v}$ continues to be $\boldsymbol{v}_{1}$.

Experimental results have shown the applicability of the Kronecker correlation model [9] to many channels. The channel state can then be represented as

$$
\boldsymbol{H}=\boldsymbol{R}^{\frac{1}{2}} \boldsymbol{H}_{w} \boldsymbol{T}^{\frac{1}{2}},
$$

where the entries of $\boldsymbol{H}_{w}$ are i. i. d. complex Gaussian $\sim \mathcal{N}_{c}(0,1)$, and $\boldsymbol{R}$ and $\boldsymbol{T}$ are the receiver and transmitter correlation matrices, respectively. While our results hold for antenna arrays with arbitrary topology, a normal distribution $\mathcal{N}\left(\theta_{r}, \sigma_{r}^{2}\right)$ is assumed for the angles of arrival (AoA) in the results presented in this paper. Here, $\theta_{r}$ is the mean AoA, and $\sigma_{r}$ is the RMS angle spread. $\theta_{r}=90^{\circ}$ corresponds to the broadside of the antenna array. We use the characterization of $\boldsymbol{R}$ given in [10].

\section{Optimal $L \times N_{r}$ Pre-Processing (TI)}

In this section, we derive the optimal channel statisticsbased $L \times N_{r}$ pre-processing matrix that maximizes the average output SNR after processing and baseband combining. Given that the pre-processor outputs $L$ streams, an explicit selection is not required. The average output SNR is given by

$$
\bar{\gamma}_{\mathrm{TI}}=\mathbf{E}_{\boldsymbol{H}}\left[\frac{\rho}{N_{t}}\left(\boldsymbol{w}^{\dagger}\left(\boldsymbol{M}_{\boldsymbol{L}} \boldsymbol{M}_{\boldsymbol{L}}^{\dagger}\right)^{-\frac{1}{2}} \boldsymbol{M}_{\boldsymbol{L}} \boldsymbol{H} \boldsymbol{v}\right)^{2}\right],
$$

where $\boldsymbol{w}$ is the baseband combining vector, $M_{L}$ is the RF pre-processing matrix, and $\mathbf{E}_{\boldsymbol{H}}$ denotes expectation over the random variable $\boldsymbol{H}$.

The following theorem characterizes the optimal solution.

Theorem 1: For a general $N_{r} \times N_{t}$ channel, $\boldsymbol{H}$, with singular value decomposition $\boldsymbol{U} \boldsymbol{\Lambda} \boldsymbol{V}^{\dagger}$, let $\boldsymbol{u}_{1}$ denote the first column of $\boldsymbol{U}$ and $\lambda_{1}$ be the largest singular value. Then, the optimal $L \times N_{r}$ pre-processing matrix, denoted by $\boldsymbol{M}_{\mathrm{TI}}$, is of the form $\boldsymbol{M}_{\mathrm{TI}}=\boldsymbol{B} \boldsymbol{Q}$, where $\boldsymbol{B}$ is any $L \times L$ full rank matrix and $\boldsymbol{Q}$ is an $L \times N_{r}$ matrix given by

$$
\boldsymbol{Q}=\left[\boldsymbol{\mu}_{1}, \boldsymbol{\mu}_{2}, \cdots, \boldsymbol{\mu}_{L}\right]^{\dagger} .
$$

Here, $\boldsymbol{\mu}_{l}$ is the singular vector of the $l^{\text {th }}$ largest singular value of the covariance matrix $\boldsymbol{R}_{\boldsymbol{u} u}=\mathbf{E}_{\boldsymbol{H}}\left[\lambda_{1}^{2} \boldsymbol{u}_{1} \boldsymbol{u}_{1}^{\dagger}\right]$. Furthermore, the optimal baseband combining vector, $\boldsymbol{w}_{\mathrm{TI}}$, is

$$
\boldsymbol{w}_{\mathrm{TI}}=\frac{\boldsymbol{Q} \boldsymbol{u}_{1}}{\left\|\boldsymbol{Q} \boldsymbol{u}_{1}\right\|} .
$$

Proof: The proof is given in Appendix A-1.

It follows from Theorem 1 that the optimal matrix, $\boldsymbol{Q}$, that maximizes the average output SNR is obtained by performing principal component analysis on $\lambda_{1} \boldsymbol{u}_{1}$ and choosing the largest $L$ principal components. This result can be seen as a generalization to MIMO systems of the single antenna correlated multipath receiver based on principal component combining [13].

In Fig. 2, we compare the behavior of TI with FFT, which employs a fixed $N_{r} \times N_{r}$ Butler matrix for pre-processing. For a uniform linear array (ULA) with $N_{r}=4$ and antenna spacing $d=0.5 \lambda$, where $\lambda$ is the wavelength, we plot the beam patterns of TI and FFT for two different channels. The mean AoA in Fig. 2(a) is $60^{\circ}$, and in Fig. 2(b) is $45^{\circ}$. We see that while TI's beam pattern adapts to the mean AoA and the angle spread (not shown in the figure) at the receiver, FFT's cannot. FFT gives optimal gains only for AoAs of $0^{\circ}, 60^{\circ}$, $90^{\circ}, 270^{\circ}$ and $300^{\circ}$.

\section{A. Phase-Only Approximation}

As mentioned, the design and fabrication of phase-only RF elements is feasible and inexpensive. This motivates us to come up with a phase-only approximation, $\boldsymbol{\Phi}$, to the optimal pre-processing solution we derived earlier. The $(i, k)^{\text {th }}$ element of $\boldsymbol{\Phi}, \phi_{i k}$, is set as the phase of the corresponding element of $\boldsymbol{M}_{\mathrm{TI}}$. In addition, each $\phi_{i k}$ can be turned off altogether using a switch $a_{i k}$. Therefore $\phi_{i k}$ is given by

$$
\phi_{i k}=a_{i k} e^{j \arg \left(M_{i k}\right)}
$$




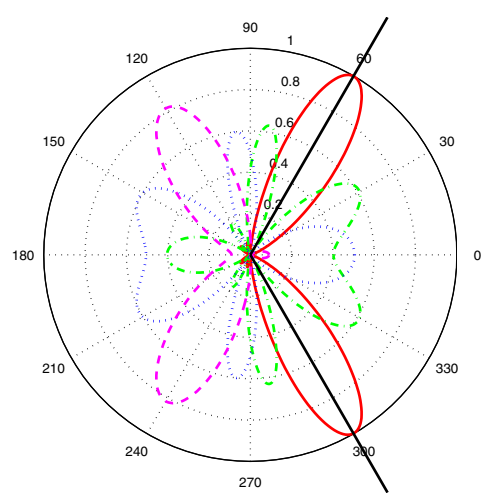

(a) Beam pattern for $M_{\mathrm{TI}}$ for $N_{r}=4, d=$ $0.5 \lambda, \theta_{r}=60^{\circ}$, and $\sigma_{r}=6^{\circ}$.

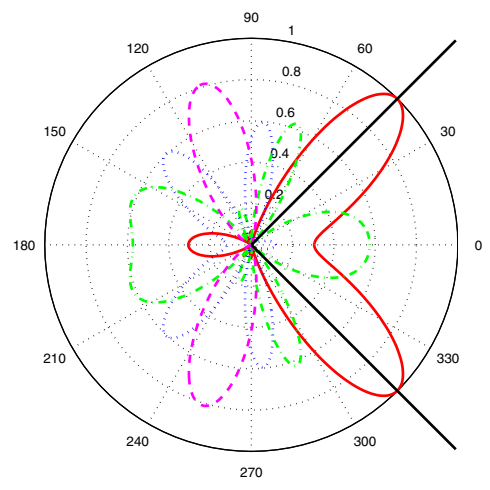

(b) Beam pattern for $M_{\mathrm{TI}}$ for $N_{r}=4, d=$ $0.5 \lambda, \theta_{r}=45^{\circ}$, and $\sigma_{r}=6^{\circ}$.

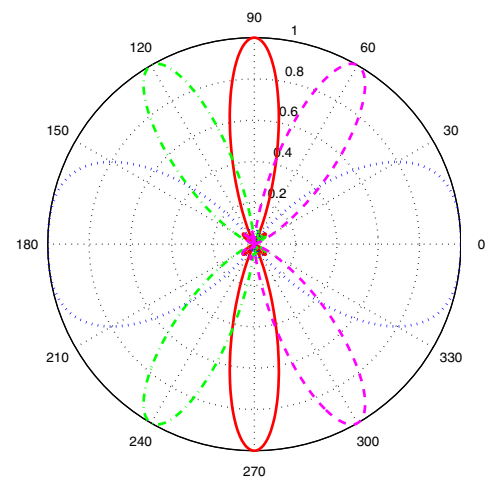

(c) Beam pattern for FFT for $N_{r}=4$ and $d=0.5 \lambda$.

Fig. 2. Beam patterns as a function of azimuth angle.

where the switch $a_{i k}$ is 0 or 1 . Let $\phi_{i}$ and $\boldsymbol{m}_{i}$ be the $i^{\text {th }}$ columns of $\boldsymbol{\Phi}$ and $\boldsymbol{M}_{\mathrm{TI}}$, respectively. While a total of $2^{N}$ combinations of the switch states are possible, the algorithm below finds the optimal switch states $a_{i j}$ that maximize the correlation between the columns of $\boldsymbol{\Phi}$ and $\boldsymbol{M}_{\mathrm{TI}}$ using only $O(L \log (L))$ computations.

1) Sort the entries of $\boldsymbol{m}_{i}$ in the descending order of absolute values, to get $m_{i[1]} \geq m_{i[2]} \geq \cdots \geq m_{i[L]}$, where $[k]$ denotes the index of the $k^{\text {th }}$ largest entry.

2) Evaluate $q_{i l}=\frac{\sum_{k=1}^{l}\left|m_{i[k]}\right|}{\sqrt{l}}, \quad 1 \leq l \leq L$.
3) Select $l_{\max }$ such that $q_{i l_{\max }}$ is maximum. ${ }^{1}$

4) Then, $a_{i k}$ is given by

$$
a_{i k}=\left\{\begin{array}{ll}
1, & \text { if } k=[1], \cdots,\left[l_{\max }\right] \\
0, & \text { otherwise }
\end{array} .\right.
$$

The additional degrees of freedom introduced by the switches $a_{i k}$ make this algorithm perform better and require fewer phase shifters than the approximation used in [6].

\section{IV. $N_{r} \times N_{r}$ Pre-Processing With Selection (TI-S)}

In this section we consider a receiver architecture, in which a channel statistics-based $N_{r} \times N_{r}$ pre-processing matrix, $\boldsymbol{M}_{\mathrm{TI}-\mathrm{S}}$, is followed by a instantaneous CSI-based selection $\boldsymbol{S}$. The output SNR is given by

$$
\begin{aligned}
\bar{\gamma}_{\mathrm{TI}-\mathrm{S}}= & \max _{\boldsymbol{M}_{\boldsymbol{N}}} \mathbf{E}_{\boldsymbol{H}}\left[\max _{\boldsymbol{S}} \frac{\rho}{N_{t}} \times\right. \\
& \left.\left(\boldsymbol{w}^{\dagger}\left(\boldsymbol{S} \boldsymbol{M}_{\boldsymbol{N}} \boldsymbol{M}_{\boldsymbol{N}}^{\dagger} \boldsymbol{S}^{\dagger}\right)^{-\frac{1}{2}} \boldsymbol{S} \boldsymbol{M}_{\boldsymbol{N}} \boldsymbol{H} \boldsymbol{v}\right)^{2}\right] .
\end{aligned}
$$

Eqn. (7) is analytically difficult to optimize because $\boldsymbol{S}$ can adapt to the instantaneous $\boldsymbol{H}$ realization. However interchanging the order of $\mathbf{E}_{\boldsymbol{H}}$ and max results in the following tractable lower bound:

$$
\begin{aligned}
\bar{\gamma}_{\text {TI-S }} \geq & \max _{\boldsymbol{S}} \max _{\boldsymbol{M}_{\boldsymbol{N}}} \mathbf{E}_{\boldsymbol{H}}\left[\frac{\rho}{N_{t}} \times\right. \\
& \left.\left(\boldsymbol{w}^{\dagger}\left(\boldsymbol{S} \boldsymbol{M}_{\boldsymbol{N}} \boldsymbol{M}_{\boldsymbol{N}}^{\dagger} \boldsymbol{S}^{\dagger}\right)^{-\frac{1}{2}} \boldsymbol{S} \boldsymbol{M}_{\boldsymbol{N}} \boldsymbol{H} \boldsymbol{v}\right)^{2}\right] .
\end{aligned}
$$

Let $C^{N, M}$ represent the set of all $N \times M$ matrices with complex entries, $C^{N}$ represent the set of all $N \times 1$ vectors with complex entries, and $U^{N, M}$ represent the set of all $N \times M$ semi-unitary matrices such that for $\boldsymbol{U} \in U^{N, M}, \boldsymbol{U} \boldsymbol{U}^{\dagger}=\boldsymbol{I}_{\boldsymbol{N}}$. Let $\boldsymbol{S}$ be a given $L \times N_{r}$ selection matrix, let $\boldsymbol{H} \in C^{N_{r}, N_{t}}$, $\mathbf{w} \in C^{L}$, and $\mathbf{v} \in C^{N_{t}}$. The following lemma shows that, without loss of optimality, we can restrict $\boldsymbol{M}_{\boldsymbol{N}}$ to be unitary.

Lemma 1:

$$
\begin{gathered}
\max _{\boldsymbol{M}_{\boldsymbol{N}} \in C^{N_{r}, N_{r}}} \mathbf{E}_{\boldsymbol{H}}\left[\frac{\rho}{N_{t}}\left(\boldsymbol{w}^{\dagger}\left(\boldsymbol{S} \boldsymbol{M}_{\boldsymbol{N}} \boldsymbol{M}_{\boldsymbol{N}}^{\dagger} \boldsymbol{S}^{\dagger}\right)^{-\frac{1}{2}} \boldsymbol{S} \boldsymbol{M}_{\boldsymbol{N}} \boldsymbol{H} \boldsymbol{v}\right)^{2}\right] \\
=\max _{\boldsymbol{U}_{\boldsymbol{N}} \in U^{N_{r}, N_{r}}} \mathbf{E}_{\boldsymbol{H}}\left[\frac{\rho}{N_{t}}\left(\boldsymbol{w}^{\dagger} \boldsymbol{S} \boldsymbol{U}_{\boldsymbol{N}} \boldsymbol{H} \boldsymbol{v}\right)^{2}\right] .
\end{gathered}
$$

Proof: Let $\boldsymbol{S} \boldsymbol{M}_{N}$ have a QR decomposition $\boldsymbol{S} \boldsymbol{M}_{N}=$ $\boldsymbol{A} \boldsymbol{U}_{L}$, where $\boldsymbol{A} \in C^{L, L}$ and $\boldsymbol{U}_{L} \in U^{L, N_{r}}$. Simplifying,

$$
\begin{aligned}
\bar{\gamma}_{\mathrm{TI}-\mathrm{S}} & =\max _{\boldsymbol{U}_{\boldsymbol{L}} \in U^{L, N_{r}}} \mathbf{E}_{\boldsymbol{H}}\left[\frac{\rho}{N_{t}}\left(\boldsymbol{w}^{\dagger} \boldsymbol{U}_{\boldsymbol{L}} \boldsymbol{H} \boldsymbol{v}\right)^{2}\right], \\
& =\max _{\boldsymbol{U}_{\boldsymbol{N}} \in U^{N_{r}, N_{r}}} \mathbf{E}_{\boldsymbol{H}}\left[\frac{\rho}{N_{t}}\left(\boldsymbol{w}^{\dagger} \boldsymbol{S} \boldsymbol{U}_{N} \boldsymbol{H} \boldsymbol{v}\right)^{2}\right] .
\end{aligned}
$$

We now state the following theorem.

\footnotetext{
${ }^{1}$ It might appear that a threshold $K$ exists such that $\theta_{i 1} \leq \theta_{i 2} \leq \cdots \leq$ $\theta_{i(K-1)} \leq \theta_{i K} \geq \theta_{i(K+1)}$ is a sufficient condition for $\theta_{i K}$ to be maximum. However, counter examples can be shown to exist.
} 
TABLE I

AVERAGE OUTPUT SNR (IN DB) FOR DIFFERENT RECEIVERS.

\begin{tabular}{|c|c|c|c|c|c|}
\hline & FC & TI-S & TI & FFT & Ant. Sel. \\
\hline$\theta_{r}=45^{\circ}, \sigma_{r}=6^{\circ}$ & 15.8 & 15.8 & 15.8 & 13.6 & 10.8 \\
\hline$\theta_{r}=60^{\circ}, \sigma_{r}=6^{\circ}$ & 15.8 & 15.8 & 15.8 & 15.8 & 10.8 \\
\hline$\theta_{r}=60^{\circ}, \sigma_{r}=15^{\circ}$ & 14.8 & 14.2 & 14.1 & 14.1 & 11.4 \\
\hline
\end{tabular}

Theorem 2: Define $U_{L}=\operatorname{span}\left\{\boldsymbol{\mu}_{1}, \boldsymbol{\mu}_{2}, \cdots, \boldsymbol{\mu}_{L}\right\}$, where $\boldsymbol{\mu}_{l}$ is as defined in Theorem 1. Let $\mathcal{N}\left(U_{L}\right)$ be the null space of $U_{L}$. Then, any $N_{r} \times N_{r}$ matrix $\boldsymbol{M}_{\mathrm{TI}-\mathrm{S}}$ of the form

$$
\boldsymbol{M}_{\mathrm{TI}-\mathrm{S}}=\boldsymbol{P}\left[\boldsymbol{\mu}_{1}, \cdots, \boldsymbol{\mu}_{L}, \boldsymbol{v}_{1}, \cdots \boldsymbol{v}_{N_{r}-L}\right]^{\dagger},
$$

maximizes (8), where $\boldsymbol{P}$ is any $N_{r} \times N_{r}$ permutation matrix and $\boldsymbol{v}_{1}, \ldots, \boldsymbol{v}_{N_{r}-L}$ form an orthonormal basis of $\mathcal{N}\left(U_{L}\right)$.

Proof: From Lemma 1, we can restrict $\boldsymbol{M}_{\mathrm{TI}-\mathrm{S}}$ to be unitary. For a given selection matrix $\boldsymbol{S}_{0}$, the problem in (8) is similar to that in Section III (with $\boldsymbol{B}=\boldsymbol{I}_{\boldsymbol{L}}$ ). It can be seen that the $\boldsymbol{M}_{N}$ that maximizes (8) has the following property: the rows of $\boldsymbol{M}_{\boldsymbol{N}}$ that are selected by $\boldsymbol{S}_{0}$ are the eigenvectors corresponding to the $L$ largest eigenvalues of $\boldsymbol{R}$. For example, if $\boldsymbol{S}_{0}=\left[\begin{array}{ll}\boldsymbol{I}_{\boldsymbol{L}} & \mathbf{0}\end{array}\right]$, then $\boldsymbol{M}_{\boldsymbol{N}}^{(\mathbf{0})}=\left[\boldsymbol{\mu}_{1}, \cdots, \boldsymbol{\mu}_{L}, \boldsymbol{v}_{1}, \cdots, \boldsymbol{v}_{N_{r}-L}\right]^{\dagger}$. Given that $\boldsymbol{M}_{\mathrm{TI}-\mathrm{S}}$ is unitary, $\boldsymbol{v}_{1}, \ldots, \boldsymbol{v}_{N_{r}-L}$ are orthonormal vectors in the null space of $U_{L}$. Any other selection matrix is a permutation of $\boldsymbol{S}_{0}$. Thus, in general, $\boldsymbol{M}_{\mathrm{TI}-\mathrm{S}}$ is a row permutation of $M_{N}^{(0)}$.

To characterize the optimal matrix $\boldsymbol{M}_{\mathrm{TI}-\mathrm{S}}$, we now identify $\boldsymbol{v}_{k}, k=1, \cdots, N_{r}-L$, such that the lower bound in (8) is improved successively. Irrespective of the choice of $\boldsymbol{v}_{k}$ 's, setting the first $L$ rows of $\boldsymbol{M}_{\mathrm{TI}-\mathrm{S}}$ to be $\left[\boldsymbol{\mu}_{1}, \cdots, \boldsymbol{\mu}_{L}\right]^{\dagger}$ ensures optimal performance if the selection matrix selects first $L$ rows. If the selection matrix selects $L+1^{\text {th }}$ row, then Theorem 1 and the constraint $\mathbf{v}_{k} \in N\left(U_{L}\right)$ imply that the average SNR is maximized when $\boldsymbol{v}_{1}=\boldsymbol{\mu}_{L+1}$. Following a similar procedure, we get $\boldsymbol{v}_{2}=\boldsymbol{\mu}_{L+2}, \ldots, \boldsymbol{v}_{N_{r}-L}=\boldsymbol{\mu}_{N_{r}}$. Thus, $\boldsymbol{M}_{\mathrm{TI}-\mathrm{S}}=\boldsymbol{P} \Upsilon^{\dagger}$, where $\boldsymbol{\Upsilon}$ is the eigenspace of $\boldsymbol{R}_{\boldsymbol{u u}}$. The details of the proof are available in [12].

\section{RESULTS}

Table I compares the average SNR of the optimal timeinvariant architectures with those previously proposed in the literature, and shows that TI/TI-S outperform antenna selection. We also look at the corresponding cumulative distribution functions (CDF), given that the CDF, unlike the average, describes the entire distribution.

Figures 3 and 4 plot the CDF of the SNR for different receiver architectures for $N_{t}=N_{r}=4$ antennas and $L=1$ demod chains. The performance of the full complexity (FC) receiver (that requires 4 demod chains) [7], FFT preprocessing receiver (followed by beam selection), conventional antenna selection, optimal $L \times N_{r}$ pre-processing (TI), phaseapproximated $L \times N_{r}$ pre-processing (TI-Ph), and $N_{r} \times N_{r}$ pre-processing followed by selection (TI-S) are compared.

In Fig. 3, the CDF of the SNR is plotted for a highly correlated channel $\left(\sigma_{r}=6^{\circ}\right)$. For a mean AoA of $60^{\circ}$, we

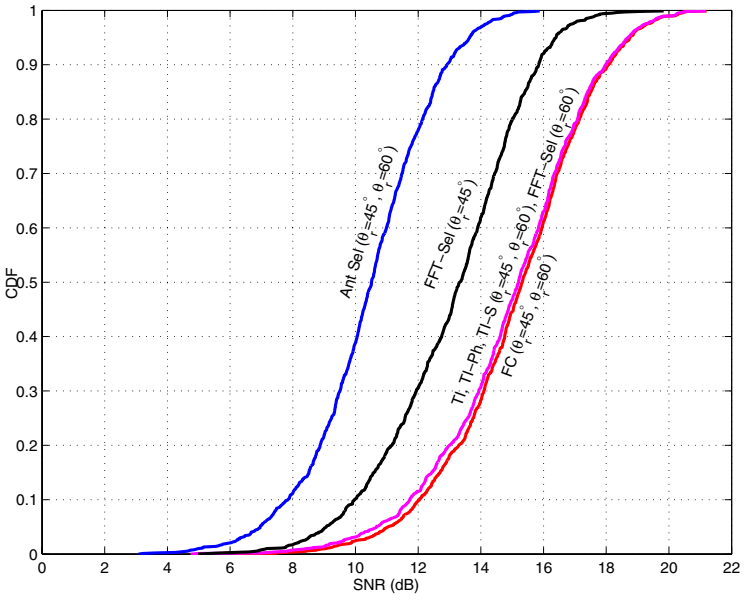

Fig. 3. CDF of the SNR for diversity system with $N_{t}=4, N_{r}=4, L=1$, $\sigma_{r}=6^{\circ}, d=0.5 \lambda, \boldsymbol{T}=\boldsymbol{I}_{\boldsymbol{N}_{\boldsymbol{t}}}$, and $\rho=10 \mathrm{~dB}$.

see that the receivers that employ RF pre-processing (TI, TI-S, and FFT), despite using the same number of demod chains, outperform conventional selection by up to $5 \mathrm{~dB}$. In fact, gains close to FC are achieved even with one demod chain. Note that these gains are achieved in TI, despite it eliminating selection altogether. TI, therefore, also provides a low complexity alternative to antenna selection algorithms [5]. When mean AoA is $45^{\circ}$, FFT performs $2 \mathrm{~dB}$ worse than TI and TI-S. This is because unlike TI and TI-S, FFT cannot adapt its beam pattern to the mean AoA; an AoA of $45^{\circ}$ falls on the minima of its beam pattern (Fig. 2(c)). Note that the phase-approximation incurs negligible performance loss; TI$\mathrm{Ph}$ comes within $0.1 \mathrm{~dB}$ of TI.

The effect of spatial correlation on the CDF of the SNR is studied in Fig. 4 by varying $\sigma_{r}$. The performance of FC receiver improves as $\sigma_{r}$ decreases, because the largest eigenvalue of the channel matrix increases with correlation. However, for conventional antenna selection, as the correlation increases, the CDF curve shifts to the left (SNR decreases) while its slope increases. TI and TI-S follow the same trend as FC. Note that the efficacy of statistics-based solutions decreases as the channel becomes less correlated. This accounts for the increasing gap between the performance of FC and channel statistics-based receivers (TI and TI-S) as $\sigma_{r}$ increases.

While the performance of TI matches TI-S for highly correlated channels, this is not so for channels with low spatial correlation. However, in such channels, TI-S still outperforms antenna selection, with performance parity occurring only for completely spatially uncorrelated channels. For further discussion and for results on multiple cluster channels, the reader is referred to [12].

\section{Conclusions}

In this paper, we proposed a novel design that combines RF pre-processing - based only on channel statistics knowledge - with selection. We derived the optimal RF pre-processing matrix and the combining vector that maximize the average 


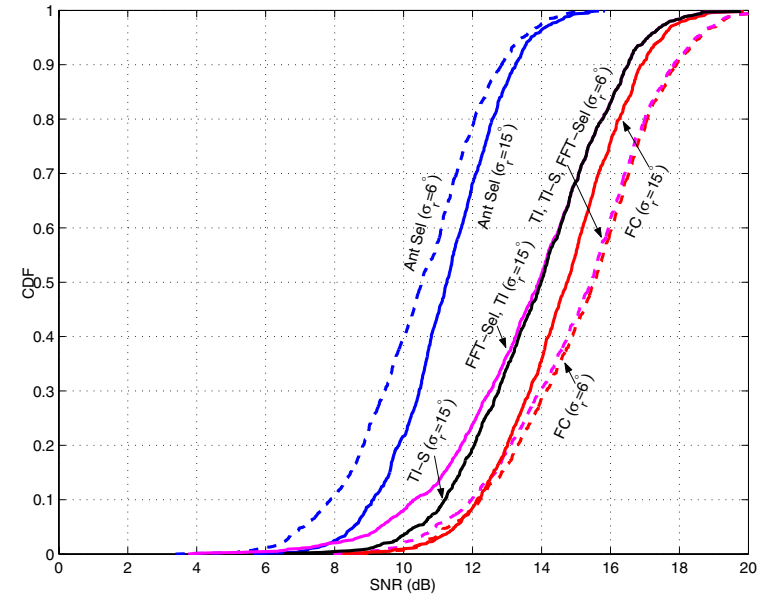

Fig. 4. Effect of spatial correlation $\sigma_{r}$ on SNR for diversity system with $N_{t}=4, N_{r}=4, L=1, \theta_{r}=60^{\circ}, d=0.5 \lambda, \boldsymbol{T}=\boldsymbol{I}_{N_{t}}$, and $\rho=10 \mathrm{~dB}$.

output SNR. We first considered a $L \times N_{r}$ pre-processing matrix that obviates the need for subsequent selection. For this case, we proved that optimal pre-processing corresponds to the $L$ largest principal components of a scaled version of the largest left singular of the channel. We then derived the optimal $N_{r} \times N_{r}$ pre-processing matrix that is followed by instantaneous CSI-based selection. This architecture outperformed the $L \times N_{r}$ scheme discussed above, especially for low spatial correlations. A phase-only approximation to the optimal solutions was also presented and was shown to incur a negligible performance loss. While this work primarily deals with receiver-side pre-processing, similar designs can be derived for the transmitter, as well.

Our results showed that, despite requiring fewer RF chains, the time-invariant solutions achieve performance close to the conventional full complexity receiver. Channel statistics-based solutions therefore provide an attractive alternative to conventional antenna selection.

\section{APPENDIX}

\section{Appendix A-1}

We first state a result in [14] that will come in handy in the proof.

Lemma 2: For any $\boldsymbol{Q} \in U^{L, N}$, given an arbitrary positive semi-definite matrix $\boldsymbol{A}$ with eigenvalues $\lambda_{1}, \lambda_{2}, \cdots, \lambda_{N}$,

$$
\sum_{k=L+1}^{N} \lambda_{k} \leq \operatorname{Tr}\left(\boldsymbol{Q} \boldsymbol{A} \boldsymbol{Q}^{\dagger}\right) \leq \sum_{k=1}^{L} \lambda_{k},
$$

where $\operatorname{Tr}(\boldsymbol{X})$ denotes trace of $\boldsymbol{X}$. Furthermore, the upper bound is achieved if and only if the columns of $\boldsymbol{Q}^{\dagger}$ are the eigenvectors associated with the $L$ largest eigenvalues of $\boldsymbol{A}$.

\section{Proof of Theorem 1}

Any $L \times N$ matrix, $\boldsymbol{M}$, with a row $\operatorname{span}^{2} L$ can be written as $\boldsymbol{M}=\boldsymbol{B Q}$, where $\boldsymbol{B}$ is a $L \times L$ full rank

\footnotetext{
${ }^{2}$ A row span less than $L$ is not considered as it is sub-optimal.
}

matrix and $\boldsymbol{Q} \in U^{L, N}$. Define $\boldsymbol{Z}=\left(\boldsymbol{M} \boldsymbol{M}^{\dagger}\right)^{-\frac{1}{2}} \boldsymbol{M}$. Then $\left\|\boldsymbol{Z}^{\dagger} \boldsymbol{w}\right\|=\|\boldsymbol{w}\|=1$. For MRT, the SNR at the receiver output is given by

$$
\gamma_{\mathrm{TI}}=\frac{\rho}{N_{t}}\left\|\lambda_{1} \boldsymbol{w}^{\dagger} \boldsymbol{Z} \boldsymbol{u}_{1}\right\|^{2} \leq \lambda_{1}^{2}\left\|\boldsymbol{Z} \boldsymbol{u}_{1}\right\|^{2},
$$

where the inequality follows from the Cauchy-Schwartz inequality. Equality in (13) is achieved if and only if $\boldsymbol{w}=\alpha \boldsymbol{Z} \boldsymbol{u}_{1}$ for some scalar $\alpha$. Therefore,

$$
\begin{aligned}
\bar{\gamma}_{\mathrm{TI}} & =\frac{\rho}{N_{t}} \mathbf{E}_{\boldsymbol{H}}\left[\lambda_{1}^{2} \boldsymbol{u}_{1}^{\dagger} \boldsymbol{Z}^{\dagger} \boldsymbol{Z} \boldsymbol{u}_{1}\right] \\
& =\frac{\rho}{N_{t}} \operatorname{Tr}\left(\boldsymbol{Q} \mathbf{E}_{\boldsymbol{H}}\left[\lambda_{1}^{2} \boldsymbol{u}_{1} \boldsymbol{u}_{1}^{\dagger}\right] \boldsymbol{Q}^{\dagger}\right), \\
& =\frac{\rho}{N_{t}} \operatorname{Tr}\left(\boldsymbol{Q} \boldsymbol{R}_{u u} \boldsymbol{Q}^{\dagger}\right) .
\end{aligned}
$$

From Lemma 2, it follows that the optimal $Q$ is obtained by choosing the $L$ largest eigenvectors of $\boldsymbol{R}_{u u}$. Note that the maximum SNR is independent of $\boldsymbol{B}$. Using the fact that $\left\|\boldsymbol{Z}^{\dagger} \boldsymbol{w}\right\|=1$, we get that $\boldsymbol{w}_{\mathrm{TI}}$ is given by

$$
\begin{gathered}
\boldsymbol{w}_{\mathrm{TI}}=\frac{\boldsymbol{Q} \boldsymbol{u}_{1}}{\left\|\boldsymbol{Q} \boldsymbol{u}_{1}\right\|} . \\
\text { REFERENCES }
\end{gathered}
$$

\section{REFERENCES}

[1] J. B. Andersen, "Antenna Arrays in Mobile Communications: Gain, Diversity, and Channel Capacity," IEEE Antennas Prop. Mag., pp. 12-16, Apr. 2000.

[2] A. F. Molisch, M. Z. Win "MIMO systems with Antenna Selection," IEEE Microwave Mag., Vol. 5, No. 1, pp. 46-56, Mar. 2004.

[3] D. A. Gore, A. Paulraj, "MIMO Antenna Subset Selection With SpaceTime Coding," IEEE Trans. Sig. Proc., pp. 2580-2588, Oct. 2002.

[4] M. Z. Win, et al., "On the SNR Penalty of MPSK with Hybrid Selection/Maximal Ratio Combining over i.i.d. Rayleigh Fading Channels," IEEE Trans. Commun., pp. 1012-1023, Jun. 2003.

[5] A. Gorokhov, D. Gore, A. Paulraj, "Receive Antenna Selection for MIMO Flat-Fading Channels: Theory and Algorithms," IEEE Trans. Inform. Theory, Vol. 49, No. 10, pp. 2687-2696, Oct. 2003.

[6] X. Zhang A. F. Molisch, S. Y. Kung, "Variable-Phase-Shift-Based RFBaseband Codesign for MIMO Antenna Selection," submitted to IEEE Trans. Sig. Proc.

[7] A. F. Molisch, X. Zhang, S. Y. Kung, J. Zhang, "FFT-Based Hybrid Antenna Selection Schemes for Spatially Correlated MIMO Channels," IEEE PIMRC, Beijing, China, Sep. 2003.

[8] T. Ohira, Y. Suzuki, H. Ogawa, H. Kamitsuna, "Megalithic Microwave Signal Processing for Phased-Array Beamforming and Steering," IEEE Trans. Microwave Theory and Techniques, pp. 2324-2332, Dec. 1997.

[9] J. P. Kermoal,et al., "A stochastic MIMO radio channel model with experimental validation," IEEE JSAC, pp. 1211-1226, Aug. 2002.

[10] D. Asztely, "On Antenna Arrays in Mobile Communication Systems: Fast Fading and GSM Base Station Receiver Algorithms," Technical Report IR-S3-SB-9611, Royal Inst. of Tech., Stockholm, Sweden, Mar. 1996.

[11] P. Sudarshan, N. B. Mehta, A. F. Molisch, J. Zhang "Channel StatisticsBased Joint RF-Baseband Design for Antenna Selection for Spatial Multiplexing," to be presented at Globecom 2004.

[12] P. Sudarshan, N. B. Mehta, A. F. Molisch, J. Zhang "Channel StatisticsBased RF Pre-Processing with Antenna Selection," submitted to IEEE Trans. Wireless Comm. 2004.

[13] M. S. Alouini, A. Scaglione, G. B. Giannakis, "PCC: Principal Components Combining for Dense Correlated Multipath Fading Environments," Proc. Veh. Technol. Conf., Vol. 5, pp. 2510-2517, Sep. 2000.

[14] P. Stoica, R. Moses, "Introduction to Spectral Analysis," Prentice-Hall, Upper Saddle River, NJ, 1997. 